\title{
Mechanical and thermal design of the CEBAF Hall a beam calorimeter*
}

\author{
M. Bevins, A. Day, P. Degtiarenko, L.A. Dillon-Townes, A. Freyberger, R. Gilman, A. Saha, \\ S. Slachtouski, Jefferson Lab, 12000 Jefferson Ave. Newport News, VA 23606, USA
}

\section{Abstract}

A calorimeter is being fabricated to provide $0.5 \%$ $1.0 \%$ absolute measurement of the beam current in the Hall A end station of the Continuous Electron Beam Accelerator Facility (CEBAF) at Jefferson Lab (JLAB). Modern powder metallurgy processes have produced high density, high thermal conductivity tungsten-copper composite materials that minimize electromagnetic and hadronic energy loss while maintaining a rapid thermal response time. Heat leaks are minimized by mounting the mass in vacuum on glass ceramic mounts. A conduction cooling scheme utilizes an advanced carbon fiber compliant thermal interface material. Transient finite difference and finite element models were developed to estimate heat leaks and thermal response times.

\section{INTRODUCTION}

An experiment scheduled for the Hall A end station of the JLAB CEBAF machine requires that absolute beam current be measured to the $0.5 \%-1.0 \%$ level for currents around $1 \mu \mathrm{A}$ (Ref. [1]). The existing beam current diagnostic devices would have uncertainties of about $30 \%$ for currents at the $1 \mu \mathrm{A}$ level. A calorimeter has been designed to improve beam current measurements (see figure 1). The calorimeter will measure the temperature rise in a metal slug after it intercepts the electron beam with well-defined energy for a well-defined time. The measured temperature rise is then used to calculate the average beam current during the exposure to the beam. Large copper and silver calorimeters built at the Stanford Linear Accelerator Center (SLAC) in the 1960's achieved precisions of about $1 \%$ (Ref. [2]).

The calorimeter is designed to contain the energy of the incident beam. Any significant energy loss compromises the ultimate accuracy. Simulations of electromagnetic and hadronic showers produced in the calorimeter show that a $16 \mathrm{~cm}$ diameter by $16 \mathrm{~cm}$ long tungsten mass with a $1 \mathrm{~cm}$ diameter by $2.5 \mathrm{~cm}$ long entrance hole would limit escaping charged and neutral energy to $0.4+/-0.2 \%$ while maintaining rapid thermal response times (Ref. [3]). To calibrate the device a cartridge heater controlled by a precision power supply is embedded into the mass.

A current measurement must be performed using only a few minutes of beam time and be able to be repeated within about 30 minutes. The calorimeter will provide precision measurements for beam powers between $0.5 \mathrm{~kW}$ $-5 \mathrm{~kW}$ at beam energies ranging from $800 \mathrm{MeV}-12 \mathrm{GeV}$.

*Work supported by the U.S. Department of Energy under contract DEAC05-84ER40150

\section{MECHANICAL DESIGN}

Pure tungsten shapes are typically produced by pressing and sintering tungsten powder followed by an extrusion or swaging operation to reduce porosity. Operations to reduce the as-sintered porosity are not practical for a part this large. An extensive search for a fully dense, high thermal conductivity, high density, tungsten composite material identified Tungstar ${ }^{\circledR}$ (Ref. [4]), a tungstencopper (95:5) powder produced by OSRAM Sylvania. This powder allows a tungsten-copper part to be made without an infiltration of copper into a sintered tungsten framework (infiltration would not be an option for a part this large). The powder is pressed then sintered producing a very dense ( $99 \%)$, homogeneous, machinable part.

Since the calorimeter must be installed upstream of the physics target, the tungsten-copper mass must be inserted into the beamline to intercept the electron beam then removed to allow normal beam operations to resume.
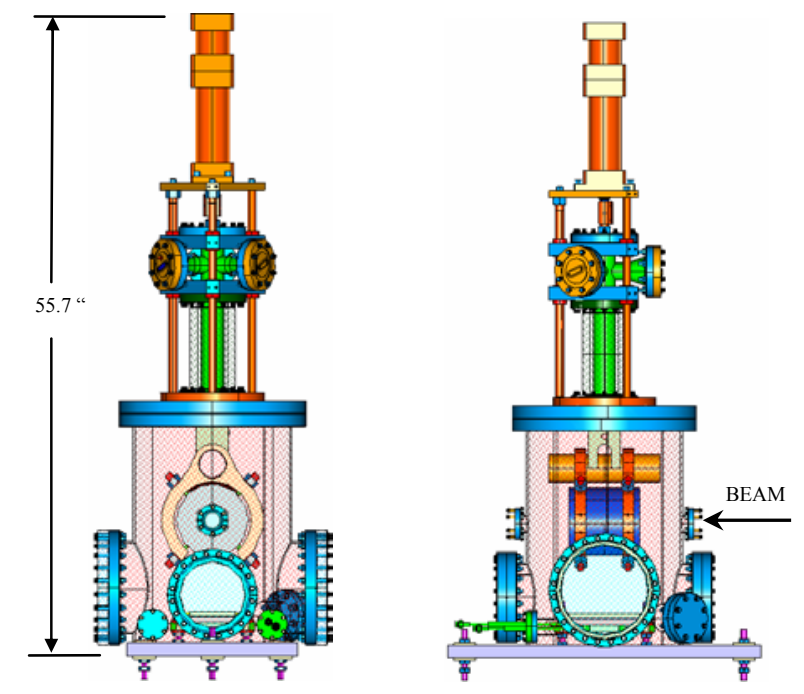

Figure 1: Hall A Calorimeter

A three position actuation scheme (using a three position air cylinder) minimizes actual beam time required to take a current measurement: 1) in-beamcharging, 2) out of beam-equilibrating, and 3) out of beam-cooling. The mass support frame incorporates an oversized beamline tube that allows beam to pass through the device in both the equilibrating and cooling positions (see figure 2).

Electrical wires for thermometry, charge bleed off, and the calibration heater are routed to the mass through 
electrical vacuum feed-throughs, then down the vertical support tube, eliminating the need for a service loop inside the vacuum chamber (see figure 2). The electrical feed through/support tube is guided using linear ball bushings and precision shafting.

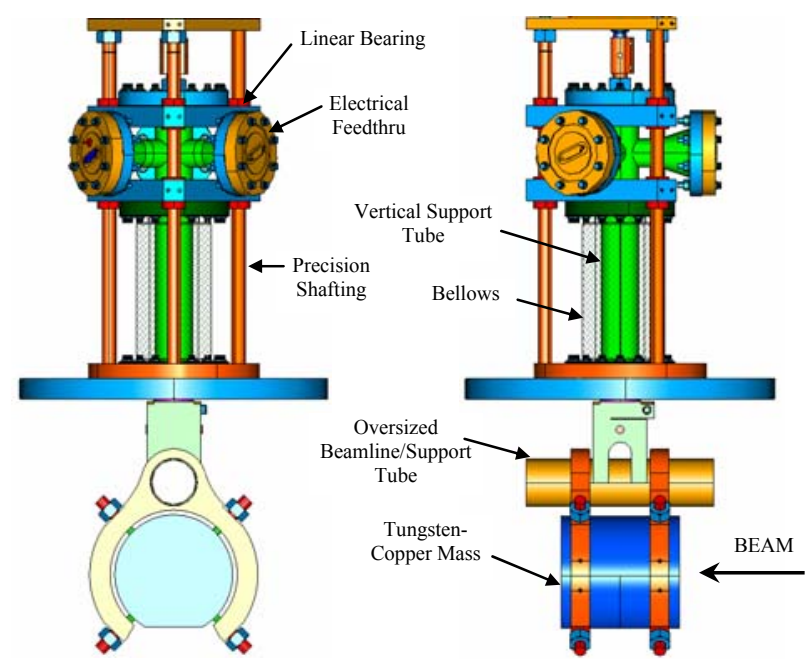

Figure 2: Mechanism

Socket set screws with glass ceramic inserts are used to position and support the mass inside the frame while providing thermal and electrical isolation. Three adjustable rods support the cooling plate foundation and allow alignment to the flat lower surface on the tungstencopper mass (see figure 3 ).

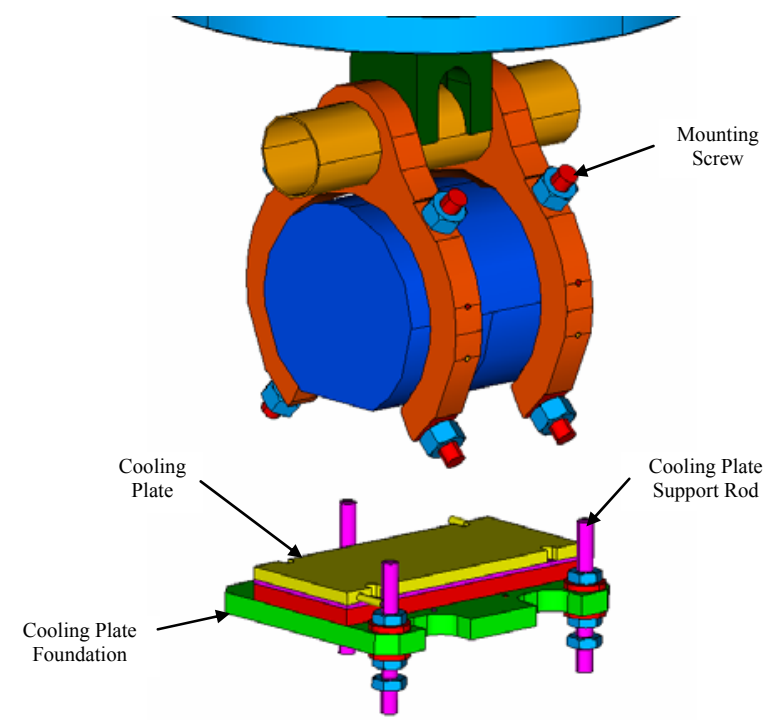

Figure 3: Mass Mount \& Cooling Plate

\section{THERMAL DESIGN}

Heat leaks to and from the mass during exposure to the beam and during equilibration must be minimized, or at least known with sufficient certainty $(<<1 \%$ of total absorbed energy).
Advanced compliant thermal interface materials with good conductance in vacuum at low interface pressures allow the mass to be cooled for subsequent measurements by bringing it in contact with a cold plate rather than embedding or otherwise attaching cooling tubes. The mass is gold coated and the vacuum vessel electropolished to reduce radiation exchange. The ceramic inserts used in the mounts minimize conductive heat transfer. The thermometry devices (RTD's) are mounted $120^{\circ}$ apart on the outer surface of the slug at each end.

\section{ANALYSIS}

Initial modeling of the thermal response time, radiative and conductive heat exchange was done using a transient two-dimensional axisymmetric implicit finite difference (FD) model written using Visual Basic for Applications in Excel. A lumped mass model that assumes minimal spatial variation in temperature was used to estimate the time required to cool the mass to repeat a measurement.

The FD model was checked using the IDEAS finite element (FE) TMG transient solver. The FE model allowed a more detailed analysis of the transient heat flow out of (and into) the tungsten-copper mass during each of the three stages of operation (i.e., charging, equilibrating, and cooling).

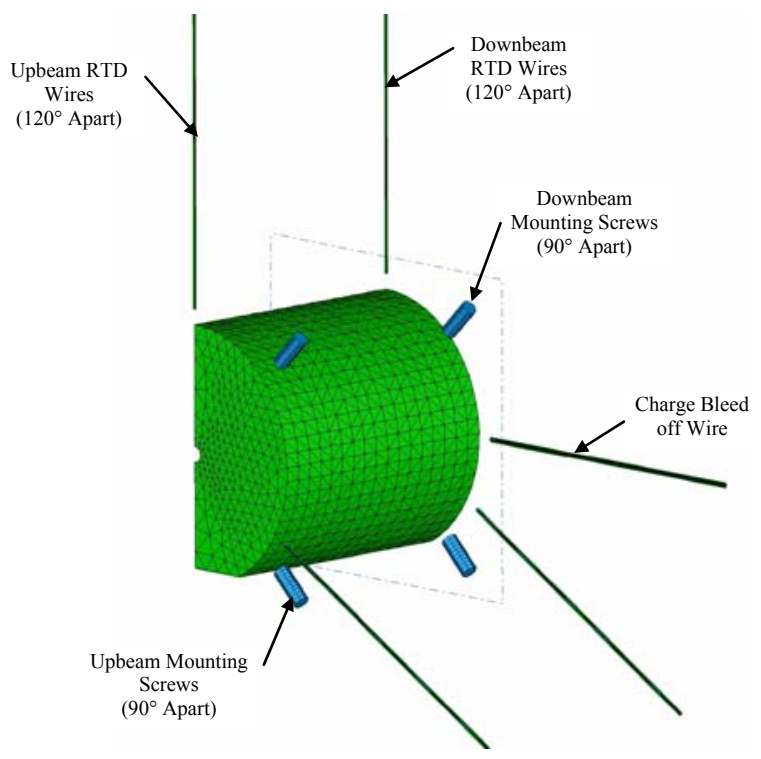

Figure 4: Finite Element Model

\section{RESULTS}

Simulations for a $48 \mathrm{sec}$ exposure to $\mathrm{I}^{*} \mathrm{E}=5 \mathrm{~kW}$ beam power are presented here. The thermal response at the RTD positions is shown in figure 5 . 


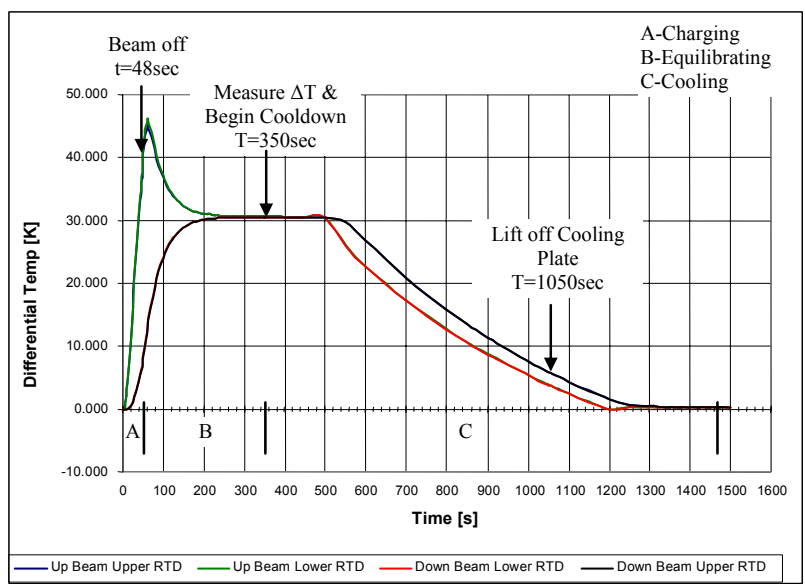

Figure 5: Thermal Response

The conductive heat leaks from the wires and mounts are shown in figure 6 .

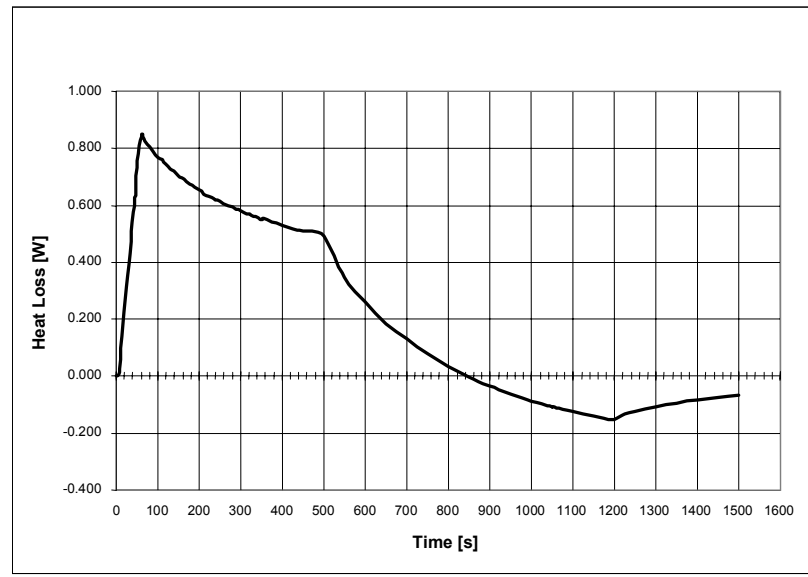

Figure 6: Conductive Heat Loss

Radiation heat losses during the charging and equilibrating phases estimated using the FD model are shown in figure 7.

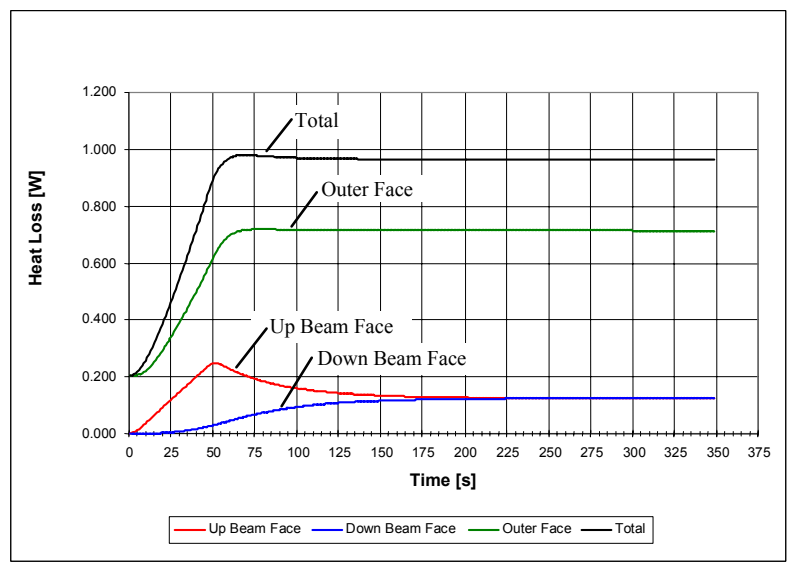

Figure 7: Radiation Heat Loss

The integrated radiation and conductive losses during the charging and equilibrating phases are combined in figure 8 .

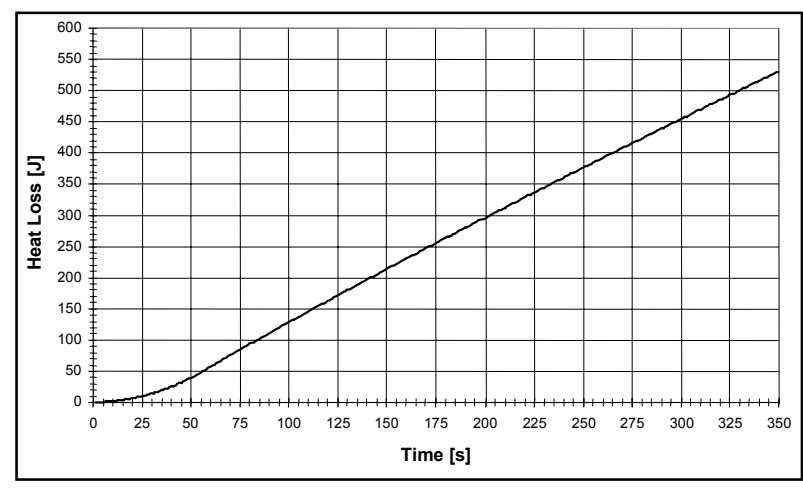

Figure 8: Integrated Total Heat Loss

The total energy deposited is $48 \mathrm{sec} * 5 \mathrm{~kW}=240 \mathrm{~kJ}$. This produces a $\Delta \mathrm{T}$ of $\sim 30 \mathrm{~K}$. Experiments performed on the thermometry instrumentation and controls show that this rise is more than sufficient to achieve the required precision (Ref. [5]). From figure 8, the total energy lost during the measurement is $531 \mathrm{~J}$. At the simulated beam power of $5 \mathrm{~kW}$, the thermal losses amount to only $0.2 \%$ of the deposited energy. The plan is to calibrate the thermal loss model with the measured test data, and then correct the beam current calibration for the calculated thermal losses.

Refinements to the FE model could include radiation exchange and a model of the heater cartridge for comparisons between simulated calibration and electron beam heating.

Thermal stress calculations are ongoing at this time. Preliminary conservative estimates show stresses high enough to warrant more refined analyses.

\section{SUMMARY}

The Hall A calorimeter thermal and mechanical design limits heat losses to the $\sim 0.2 \%$ level. The design minimizes actual beam time required to take a measurement and allows a measurement to be repeated within $\sim 20 \mathrm{~min}$.

The device is currently in fabrication with initial bench testing (using the heater) expected to begin this summer. Installation into the Hall is planned for early 2006.

\section{REFERENCES}

[1] R. Gilman, "Hall A Beam Calorimeter: Overview, Specifications, Operations", JLAB internal document, May 6, 2004

[2] G.E. Fischer and Y. Murata, "A Beam Monitor System for High-Intensity Photon Beams in the Multi-GeV Range", NIM volume 78, pages 25-39, 1970, SLAC-PUB-0605

[3] P. Degtiarenko, "Tungsten Calorimeter Model Calculations and Radiation Issues", JLAB internal document, May 14, 2004

[4] Tungstar ${ }^{\circledR}$ is a registered trademark of OSRAM Sylvania, http://www.sylvania.com

[5] A. Freyberger, JLAB internal communication 